\title{
ESTIMATES FOR EIGENFUNCTIONS AND EIGENVALUES OF NONLINEAR ELLIPTIC PROBLEMS
}

BY

\author{
CHRIS COSNER
}

\begin{abstract}
We consider solutions to the nonlinear eigenvalue problem
(*) $\quad A(x, \vec{u}) \vec{u}+\lambda f(x, \vec{u})=0 \quad$ in $\Omega, \quad \vec{u}=0 \quad$ on $\partial \Omega, \quad \vec{u} \not \equiv 0$,

where $(*)$ is a quasilinear strongly coupled second order elliptic system of partial differential equations and $\Omega \subseteq \mathbf{R}^{n}$ is a smooth bounded domain. We obtain lower bounds for $\lambda$ in the case where $f(x, \vec{u})$ has linear growth, and relations between $\lambda, \Omega$, and esssup $|\vec{u}|$ when $f(x, \vec{u})$ has sub- or superlinear growth. The estimates are based on integration by parts and application of certain Sobolev inequalities. We briefly discuss extensions to higher order systems.
\end{abstract}

1. Introduction. A classical problem of mathematical physics is to estimate the eigenvalues of linear elliptic operators. In recent years considerable attention has been given to nonlinear elliptic eigenvalue problems. Such problems also arise in applications, for example in the analysis of steady states for reaction-diffusion equations. The object of this paper is to obtain estimates relating the eigenvalues and eigenfunctions of nonlinear elliptic problems. In the linear case elliptic operators on bounded domains typically have a discrete set of eigenvalues which can be shown to satisfy various bounds. Such bounds have been obtained by many investigators, notably Faber [11], Krahn [19, 20], Barta [7], Weinberger [38], Payne and Weinberger [26], Protter [29-31], Hersch [18], Protter and Weinberger [32] and Fichera [12], among many others. There is a close relationship between such estimates and isoperimetric inequalities; this is discussed in Pólya and Szegö [28], Payne [24] and Bandle [6]. In the linear case it is not possible to give direct bounds on eigenfunctions, since any multiple of an eigenfunction is also an eigenfunction for the same eigenvalue. In the nonlinear case the situation is different. For the equation $\Delta u=$ $\lambda u^{p}=0$ with Dirichlet boundary conditions on a bounded domain, it was shown by Levinson [22] that there can be solutions for all $\lambda>0$; furthermore, results of Ambrosetti and Rabinowitz [4] imply there may be infinitely many solutions for fixed choices of $p$ and $\lambda$. However, there may be relationships between the eigenvalue $\lambda$ and the size of the eigenfunctions associated with $\lambda$. Some results in that direction have been obtained by Schaefer and Sperb [33], Payne and Stakgold, Bandle, and others; see [35].

In this article relationships between eigenvalues and eigenfunctions are obtained for fairly general second order systems. The method of estimation is based on

Received by the editors November 17, 1982.

1980 Mathematics Subject Classification. Primary 35P15, 35J60, 35J65, 35B30.

(C) 1984 American Mathematical Society $0002-9947 / 84 \$ 1.00+\$ .25$ per page 
integration by parts and application of the Sobolev inequalities. It is interesting to note that the well-known Faber-Krahn inequality for the first eigenvalue of the Laplacian $[\mathbf{1 1}, \mathbf{1 9}, \mathbf{2 0}]$ is based on the idea of symmetrization; so is the inequality obtained by Talenti [36] for the optimal constant in certain Sobolev inequalities. The results are both related to isoperimetric inequalities, which are discussed in $[5,23,24$, 28]. The relationship between the Faber-Krahn and Sobolev inequalities is discussed in [23]. Although isoperimetric inequalities are not used directly in this paper, both the Faber-Krahn estimate and Talenti's bound for the Sobolev embedding are used, so the results are related to the traditional isoperimetric methods for estimating eigenvalues.

The systems considered here mostly have the form

$$
\begin{aligned}
\sum_{\beta=1}^{m}\left[\sum_{i, j=1}^{n}\left(a_{i j}^{\alpha \beta}(x, \vec{u}) u_{x_{i}}^{\beta}\right)_{x_{j}}+\sum_{i=1}^{n} b_{i}^{\alpha \beta}(x, \vec{u}) u_{x_{i}}^{\beta}+c^{\alpha \beta}(x, \vec{u}) u^{\beta}\right] \\
+\lambda f^{\alpha}(x, \vec{u})=0 \text { in } \Omega, \quad \alpha=1, \ldots, m, \quad \vec{u}=0 \text { on } \partial \Omega, \quad \vec{u} \not \equiv 0 \text { in } \Omega,
\end{aligned}
$$

where $\vec{u}=\left(u^{1}, \ldots, u^{m}\right)$ and $\Omega \subseteq \mathbf{R}^{n}$ is a smooth bounded domain. A brief discussion is given of the higher order analog of (1.1). The system (1.1) is strongly coupled, that is, there is coupling in the second order terms; it is not, in general, even formally selfadjoint. An ellipticity condition is assumed, namely that

$$
\sum_{\alpha, \beta=1}^{m} \sum_{i, j=1}^{n} a_{i j}^{\alpha \beta} \xi_{j}^{\alpha} \xi_{i}^{\beta} \geqslant a_{0}|\xi|^{2} \quad \text { for all } x \in \Omega, \vec{u} \in \mathbf{R}^{m}, \vec{\xi} \in \mathbf{R}^{m n}
$$

A growth condition also is imposed on $f=\left(f^{1}, \ldots, f^{m}\right)$, namely that for some constants $f_{0}, q>0$,

$$
|\vec{u} \cdot f(x, \vec{u})| \leqslant f_{0}|\vec{u}|^{1+q} \quad \text { for all } x \in \Omega, \vec{u} \in \mathbf{R}^{m} .
$$

Other conditions are imposed for some results. Existence theorems for equations of various forms allowed by (1.1)-(1.3) have been discussed by many authors, including Levinson [22], Pohozaev [27], Browder [9], Berger [8], Kramer [21], Ambrosetti and Rabinowitz [4], Amann and Crandall [3] and De Figueiredo, Lions and Nussbaum [10]. Most of the existence results require additional conditions on (1.1), or sign conditions on $\vec{u}$ or $\vec{f}$, but together they cover many types of problems with the general form (1.1).

The main results of this article are obtained in $\$ 3$. They fall into three cases, depending on the growth of $\vec{f}$. If $n>2$ and the constant $q$ satisfies $1<q \leqslant n+$ $2 /(n-2)$, then we obtain lower bounds for essup $|\vec{u}|$ in terms of $|\lambda|$ and the $n$-volume of $\Omega$. (For $n=2$, we only need $q>1$.) The bounds require either an additional coercivity condition on (1.1) (which is satisfied in the case $b_{i}^{\alpha \beta} \equiv c^{\alpha \beta} \equiv 0$ ) or that $\Omega$ have sufficiently small volume. In fact, all results have that restriction. Some similar results have been obtained by Schaefer and Sperb [33] for a single, more specialized equation; related work has been done by Payne, Stakgold, Bandle and others; see [5, 6, 25] and especially the book by Sperb [35]. Those results typically depend on the maximum principle or on other special features of the 
equation which (1.1), in general, does not have. However, the growth restrictions on $\vec{f}$ may be weaker; see [5 and 35]. For positive solutions upper bounds have been obtained by Gidas [14], Gidas and Spruck [16], and De Figueiredo, Lions and Nussbaum [10]. Those bounds are based on arguments involving the maximum principle and a type of symmetrization developed by Gidas, Ni and Nirenberg [15]. In special cases of (1.1) such bounds apply; however, there are no positivity assumptions in (1.1) and the system need not satisfy a maximum principle. Upper bounds for positive solutions of a single equation in $\Omega \nsubseteq \mathbf{R}^{2}$ were obtained by Turner [37].

If $q=1$ in (1.3) then the function $\vec{f}$ has linear growth. In that case an estimate from below is obtained for $|\lambda|$ in terms of the first eigenvalue of the Laplacian on $\Omega$. The results extend to the case where $\lambda$ is complex, and even to the case where the last term of $(1.1)$ is replaced by $\lambda^{\alpha} f^{\alpha}(x, \vec{u})$ with possibly different constants in each equation. Thus, since (1.1) is strongly coupled, some extensions of results due to Protter [31] are obtained.

If $0 \leqslant q<1$ in (1.3) then upper bounds for ess sup $|\vec{u}|$ are obtained in terms of $|\lambda|$. Such bounds are not as sharp as those found for $q \geqslant 1$. Similar results have been obtained for more specialized equations by Schaefer and Sperb [33]; results with more specialized structure for the equation, but weaker or different growth conditions on $\vec{f}$, are obtained in $[5,14,16,37]$.

Similar results can be obtained in the case of higher order systems; this is discussed in $\$ 4$.

The methods used here do not always yield especially sharp results; the main new feature is that they require no sign or monotonicity conditions on $\vec{u}$ or $\vec{f}$, no selfadjointness conditions on the left side of (1.1), and do not depend on the maximum principle. Thus they apply in considerable generality.

2. Notation and basic inequalities. Let $\Omega \subseteq \mathbf{R}^{n}$ be a bounded domain with smooth boundary. The $n$-volume of $\Omega$ will be denoted by $|\Omega|$. In what follows, all integrals are taken over $\Omega$. The usual Sobolev spaces of functions with $k$ weak derivatives in $L^{p}$ are denoted by $W^{k, p}$; the completion of $C_{0}^{\infty}(\Omega)$ in $W^{k, p}$ by $W_{0}^{k, p}$. The norm in $W^{k, p}$ for a single function is defined in the usual way (see [1 or 17]) and denoted by \|\|$_{k, p}$. We also consider vector functions $\vec{u}: \Omega \rightarrow \mathbf{R}^{m}$ for which we define the $L^{p}$ norm by

$$
\|\vec{u}\|_{0, p}=\left[\int|\vec{u}|^{p} d x\right]^{1 / p},
$$

and then define the Sobolev norms in the usual way.

We will need various inequalities relating the $L^{p}$ norms of functions and their derivatives. We start by discussing a single function. The first inequality is the well-known one of Faber and Krahn: if $\Omega$ is bounded, then, for $u \in W_{0}^{1,2}$,

$$
j_{(n-2) / 2}^{2}\left(\frac{\omega_{n}}{|\Omega|}\right)^{2 / n} \int|u|^{2} d x \leqslant \mu_{1}(\Omega) \int|u|^{2} d x \leqslant \int|\nabla u|^{2} d x,
$$


where $\mu_{1}(\Omega)>0$ is the first eigenvalue for

$$
\Delta u+\mu u=0 \quad \text { on } \Omega, \quad u=0 \quad \text { on } \partial \Omega,
$$

$\omega_{n}=2 \pi^{n / 2} / n \Gamma\left(\frac{n}{2}\right)$ is the $n$-volume of the unit sphere in $\mathbf{R}^{n}$, and $j_{(n-2) / 2}$ is the first zero of the Bessel function $J_{(n-2) / 2}(x)$. Inequality (2.2) is discussed in $[6,23,24,28$, 33 ] and many other references; it was proved in [11, 19 and 20]. The inequality may be obtained via the method of symmetrization. Related methods are also used in [36] to obtain results including the following special case of the Sobolev inequality: if $n>2, p=p^{*}(n)=2 n /(n-2)$, then for $u \in W_{0}^{1,2}$,

$$
\|u\|_{0, p}^{2} \leqslant C(n)^{2} \int|\nabla u|^{2} d x
$$

where the optimum value (for arbitrary domain) of $C(n)$ is

$$
C(n)=\frac{1}{\pi^{1 / 2} n^{1 / 2}(n-2)^{1 / 2}}\left(\frac{\Gamma(n)}{\Gamma(n / 2)}\right)^{1 / n}=\frac{1}{\pi n^{1 / 2}(n-2)^{1 / 2}}\left(\frac{n ! \omega_{n}}{2}\right)^{1 / n} .
$$

Inequalities (2.4) and (2.5) are also discussed in [17]. A great deal of work has been done on inequalities of types (2.2) and (2.4); for discussion see [6, 23, 24, 28]. Inequalities (2.2) and (2.4) give bounds for $\|u\|_{0, p}$ when $p=2$ and $p=p^{*}(n)=$ $2 n /(n-2)$; for other values of $p$ we will need the interpolation inequalities

$$
\|u\|_{0, r} \leqslant\|u\|_{0, p}^{\delta}\|u\|_{0, q}^{1-\delta} \text { for } p \leqslant q \leqslant r, 1 / r=\delta / p+(1-\delta) q
$$

and

$$
\|u\|_{0, q} \leqslant|\Omega|^{1 / q-1 / p}\|u\|_{0, p} \text { for } p \geqslant q \geqslant 1 .
$$

In the case $2 \leqslant p \leqslant p^{*}(n)$, we can combine inequalities (2.2), (2.4) and (2.6) to obtain

$$
\begin{aligned}
\|u\|_{0, p}^{2} & \leqslant C(n)^{2(1-\delta)} \mu_{1}(\Omega)^{-\delta} \int|\nabla u|^{2} d x \\
& \leqslant \frac{C(n)^{2(1-\delta)}}{\left(j_{(n-2) / 2} \omega_{n}^{1 / n}\right)^{2 \delta}}|\Omega|^{2 \delta / n} \int|\nabla u|^{2} d x
\end{aligned}
$$

where $\delta=2\left(p^{*}(n)-p\right) / p\left(p^{*}(n)-2\right)$. Combining (2.2) and (2.7) yields, for $1 \leqslant p$ $\leqslant 2$,

$$
\|u\|_{0, p}^{2} \leqslant \mu_{1}(\Omega)^{-1}|\Omega|^{(2-p) / p} \int|\nabla u|^{2} d x
$$

Finally, we have the Sobolev embedding theorems (see [1]):

if $u \in W^{k, p}$ and $k p<n$, then for $p \leqslant q \leqslant n p /(n-k p)$,

$$
\|u\|_{0, q} \leqslant M_{0}(n, \Omega, k, p, q)\|u\|_{k, p} ;
$$

if $k p=n$, for all finite $q \geqslant p$ we have

$$
\|u\|_{0, q} \leqslant M_{1}(n, \Omega, k, q)\|u\|_{k, p} ;
$$


and if $k p>n$ we have

$$
\sup |u| \leqslant M_{2}(n, \Omega, k, p)\|u\|_{k, p} .
$$

If $u \in W_{0}^{k, p}$ then the constants in (2.10)-(2.12) may be taken with no dependence on $\Omega$. (This independence of $\Omega$ follows because for $u \in W_{0}^{k, p}$ we can extend $u$ to $\mathbf{R}^{n}$ by taking $u=0$ outside $\Omega$; that extension isometrically embeds $W_{0}^{k, p}(\Omega)$ in $W^{k, p}\left(\mathbf{R}^{n}\right)$, and we can then apply the Sobolev embedding theorem in $\mathbf{R}^{n}$.) We can eliminate the restriction $q \geqslant p$ in (2.10), (2.11) by using (2.7) as long as $q \geqslant 1$; however, even if $u \in W_{0}^{k, p}$, we will then have constants depending on $\Omega$.

So far we have only discussed the case where $u$ is a single function. Similar estimates still hold for $\vec{u}=\left(u^{1}, \ldots, u^{m}\right)$ with minor modifications. Since we will ultimately wish to estimate $|\vec{u}|$, we define $\|\vec{u}\|_{0, p}$ via (2.1). However, (2.8) and (2.9), if applied to $u$, give bounds not on $\|\vec{u}\|_{0, p}^{2}$, as defined in (2.1); but rather on $\sum_{\alpha=1}^{m}\left\|u^{\alpha}\right\|_{0, p}^{2}$. To extend the results to $u$ we require the following inequality:

$$
\|\vec{u}\|_{0, p}^{2} \leqslant m^{(p-2) / p} \sum_{\alpha=1}^{m}\left\|u^{\alpha}\right\|_{0, p}^{2} \quad \text { for } p \geqslant 2 .
$$

To obtain (2.13) we note that

$$
\|\vec{u}\|_{0, p}^{2}=\left[\int\left(\sum_{\alpha=1}^{m}\left|u^{\alpha}\right|^{2}\right)^{p / 2} d x\right]^{2 / p} .
$$

By Hölder's inequality applied to the sum,

$$
|\vec{u}|^{2}=\sum_{\alpha=1}^{m}\left|u^{\alpha}\right|^{2} \leqslant m^{(p-2) / p}\left(\sum_{\alpha=1}^{m}\left|u^{\alpha}\right|^{p}\right)^{2 / p},
$$

so

$$
\|\vec{u}\|_{0, p}^{2} \leqslant m^{(p-2) / p}\left(\sum_{\alpha=1}^{m} \int\left|u^{\alpha}\right|^{p} d x\right)^{2 / p} .
$$

Since $p \geqslant 2$

$$
\left(\sum_{\alpha=1}^{m} V^{\alpha}\right)^{2 / p} \leqslant \sum_{\alpha=1}^{m}\left(V^{\alpha}\right)^{2 / p} \text { for } V^{\alpha} \geqslant 0
$$

so (2.13) follows.

If $1 \leqslant p<2$, then the above analysis fails, but since all norms on $\mathbf{R}^{m}$ are equivalent, we still have, for some constant $B(m)$, the inequality

$$
\|\vec{u}\|_{0, p}^{2} \leqslant B(m) \sum_{\alpha=1}^{m}\left\|u^{\alpha}\right\|_{0, p}^{2} .
$$

(We can take $B(m)=m^{3}$, but that is probably not optimal.)

Similarly, (2.10), (2.11) and (2.13) extend to the case $m>^{*} 1$ with the introduction of a factor depending on $m$ in the constant. 
3. Estimates for second order problems. We consider nontrivial solutions $\vec{u}=$ $\left(u^{1}, \ldots, u^{m}\right) \in W_{0}^{1,2} \cap L^{\infty}$ of equations of the form

$$
\begin{aligned}
& \sum_{\beta=1}^{m}\left[\sum_{i, j=1}^{n}\left(a_{i j}^{\alpha \beta}(x, \vec{u}) u_{x_{i}}^{\beta}\right)_{x_{j}}+\sum_{i=1}^{n} b_{i}^{\alpha \beta}(x, \vec{u}) u_{x_{i}}^{\beta}+c^{\alpha \beta}(x, \vec{u}) u^{\beta}\right] \\
& +\lambda f^{\alpha}(x, \vec{u})=0 \text { in } \Omega, \quad \alpha=1, \ldots, m, \quad \vec{u}=0 \text { on } \partial \Omega, \quad \vec{u} \neq \equiv \text { in } \Omega,
\end{aligned}
$$

where $\lambda$ is a parameter. For the sake of simplicity we state the results for the case where $\vec{u}$ is real and $\lambda$ is a single real parameter, but most of the results extend with minor changes to the case where $\vec{u}$ and $\lambda$ may be complex, and/or the term $\lambda f^{\alpha}$ in (3.1) is replaced by $\lambda^{\alpha} f^{\alpha}$ with a different $\lambda^{\alpha}$ in each equation. (See the remarks following Lemma 1 for further discussion.)

We require that $\vec{u}$ be a solution to (3.1) in the usual weak sense, that is, $\vec{u} \in W_{0}^{1,2} \cap L^{\infty}$, and, for test functions $\vec{v} \in W^{1,2}$, the integral equation obtained by taking the scalar product of (3.1) by $\vec{v}$ and formally integrating the second order terms by parts be satisfied. We always assume the coefficients of (3.1) are bounded and measurable and $\vec{f}(x, u)=\left(f^{1}(x, \vec{u}), \ldots, f^{m}(x, \vec{u})\right)$ is measurable and bounded in $x \in \Omega$ for any fixed $\vec{u}$. We also assume there is a constant $a_{0}>0$ such that, for all $\vec{\xi}=\left(\xi_{i}^{\alpha}\right), \alpha=1, \ldots, m, i=1, \ldots, n$ in $\mathbf{R}^{m n}$, all $x \in \Omega$, and all $\vec{u} \in \mathbf{R}^{m}$,

$$
\sum_{\alpha, \beta=1}^{m} \sum_{i, j=1}^{n} a_{i j}^{\alpha \beta}(x, \vec{u}) \xi_{j}^{\alpha} \xi_{i}^{\beta} \geqslant a_{0}|\vec{\xi}|^{2} .
$$

Condition (3.2) implies that (3.1) is elliptic. Observe that other than (3.2) we have imposed no sign or symmetry conditions on (3.1).

If the coefficients $b_{i}^{\alpha \beta}$ and $c^{\alpha \beta}$ are not all identically zero, we sometimes impose an additional condition on the coefficients, namely that there exists a constant $a_{1}>0$ such that for $\vec{\xi} \in \mathbf{R}^{m n}, \vec{\eta} \in \mathbf{R}^{n}$, and all $x \in \Omega, \vec{u} \in \mathbf{R}^{m}$, we have

$$
\sum_{\alpha, \beta=1}^{m}\left[\sum_{i, j=1}^{n} a_{i j}^{\alpha \beta} \xi_{j}^{\alpha} \xi_{i}^{\beta}-\sum_{i=1}^{n} b_{i}^{\alpha \beta} \xi_{i}^{\beta} \eta^{\alpha}-c^{\alpha \beta} \eta^{\alpha} \eta^{\beta}\right] \geqslant a_{1}|\vec{\xi}|^{2} .
$$

If $b_{i}^{\alpha \beta} \equiv c^{\alpha \beta} \equiv 0$ then (3.3) reduces to (3.2). In general, there are simple conditions which guarantee (3.3). For example, suppose the matrix $c^{\alpha \beta}$ is negative definite, so

$$
\sum_{\alpha, \beta=1}^{m} c^{\alpha \beta} \eta^{\alpha} \eta^{\beta} \leqslant-c_{0}|\vec{\eta}|^{2} \quad \text { for some } c_{0}>0
$$

and

$$
\sum_{\alpha, \beta=1}^{m} \sum_{i=1}^{n} b_{i}^{\alpha \beta} \xi_{i}^{\beta} \eta^{\alpha} \leqslant b_{0}|\vec{\xi} \| \vec{\eta}| \text { for some constant } b_{0} .
$$

Then the left side of (3.3) is bounded below by $a_{0}|\vec{\xi}|^{2}-b_{0}|\vec{\xi}||\vec{\eta}|+c_{0}|\vec{\eta}|^{2}$, which is bounded below by $a_{1}\left(|\vec{\xi}|^{2}+|\vec{\eta}|^{2}\right) \leqslant a_{1}|\vec{\xi}|^{2}$ for some $a_{1}>0$ provided $4 a_{0} c_{0}>b_{0}^{2}$. Condition (3.3) may also occur if the matrices $b_{i}^{\alpha \beta}, c^{\alpha \beta}$ are zero for $\alpha, \beta \geqslant k$ with $k<m$, and the $k \times k$ nonzero blocks satisfy the appropriate definiteness and size conditions. 
The main conditions we impose on $\vec{f}(x, \vec{u})$, are growth conditions; if there is no sign condition on $\vec{u} \cdot \vec{f}(x, \vec{u})$ we require

$$
|\vec{u} \cdot \vec{f}(x, \vec{u})| \leqslant f_{0}|\vec{u}|^{1+q},
$$

where $f_{0}>0$ is a constant and $0 \leqslant q \leqslant q^{*}(n)$, where $q^{*}(n)=p^{*}(n)-1=$ $(n+2) /(n-2)$, for $n>2$, with $0 \leqslant q<\infty$ for $n=2$. In some cases we can replace condition (3.4) with the one-sided bound

$$
\vec{u} \cdot \vec{f}(x, \vec{u}) \leqslant f_{0}|\vec{u}|^{1+q}
$$

with $f_{0}, q$ as in (3.4).

We can now state the fundamental lemmas of the paper.

LEMma 1. Suppose $\vec{u}$ satisfies (3.1) in $\Omega \subseteq \mathbf{R}^{n}, n>2$, with $\Omega$ bounded. Assume (3.3) and (3.4) hold with the constant $q$ in (3.4) satisfying $1 \leqslant q \leqslant(n+2) /(n-2)$. Then $\vec{u}$ satisfies the inequality

$$
\begin{aligned}
1 & \leqslant|\lambda| f_{0} a_{1}^{-1} m^{(p-2) / p} C(n)^{2(1-\delta)} \mu_{1}(\Omega)^{-\delta}\|\vec{u}\|_{0, p}^{p-2} \\
& \leqslant|\lambda| f_{0} a_{1}^{-1} m^{(p-2) / p} C(n)^{2(1-\delta)}\left(j_{(n-2) / n} \omega_{n}^{1 / n}\right)^{-2 \delta}|\Omega|^{2 \delta / n}\|\vec{u}\|_{0, p}^{p-2},
\end{aligned}
$$

where $p=q+1 \geqslant 2, \delta=2\left(p^{*}(n)-p\right) / p\left(p^{*}(n)-2\right)$ with $p^{*}(n)=2 n /(n-2)$, and the other constants are as in $\S 2$.

Proof. By our definition of a weak solution to (3.1), $\vec{u}$ satisfies

$$
\int \sum_{\alpha, \beta=1}^{m}\left[\sum_{i, j=1}^{n} a_{i j}^{\alpha \beta} u_{x_{j}}^{\alpha} u_{x_{i}}^{\beta}-\sum_{i=1}^{n} b_{i}^{\alpha \beta} u^{\alpha} u_{x_{i}}^{\beta}-c^{\alpha \beta} u^{\alpha} u^{\beta}\right]=\lambda \int \vec{u} \cdot \vec{f}(x, \vec{u}) .
$$

Applying (3.3) and (3.4) yields

$$
a_{1} \sum_{\alpha=1}^{m} \int\left|\nabla u^{\alpha}\right|^{2} \leqslant|\lambda| f_{0}\|\vec{u}\|_{0, p}^{p}
$$

where $p=1+q$. From (2.8) we have

$$
\sum_{\alpha=1}^{m}\left\|u^{\alpha}\right\|_{0, p}^{2} \leqslant|\lambda| f_{0} a_{1}^{-1} C(n)^{2(1-\delta)} \mu_{1}(\Omega)^{-\delta}\|\vec{u}\|_{0, p}^{p},
$$

where $\delta=2\left(p^{*}(n)-p\right) / p\left(p^{*}(n)-2\right), p^{*}(n)=2 n /(n-2)$. Combining (3.9) with (2.13) yields

$$
\|\vec{u}\|_{0, p}^{2} \leqslant|\lambda| f_{0} a_{1}^{-1} m^{(p-2) / p} C(n)^{2(1-\delta)} \mu_{1}(\Omega)^{-\delta}\|\vec{u}\|_{0, p}^{p}
$$

which immediately yields (3.6). (The second inequality in (3.6) is just the Faber-Krahn bound for $\mu_{1}(\Omega)$ applied to the first inquality.)

REMARKS. (1) If $\vec{u} \cdot \vec{f}(x, \vec{u}) \geqslant 0$ then, by (3.7), $\lambda \geqslant 0$, so we may replace (3.4) with (3.5) and replace $|\lambda|$ with $\lambda$ in (3.6). In many cases there is special interest in positive solutions of $(3.1)$; see $[10,14,15,16,22,25,37]$, among others. If we require $u^{\alpha} \geqslant 0$ then (3.5) need only hold under that condition.

(2) We have assumed $\vec{u}$ and $\lambda$ are real. If we define $\vec{u} \cdot \vec{v}=\sum_{\alpha=1}^{m} \vec{u}^{\alpha} v^{\alpha}$ and impose certain symmetry conditions on (3.1), then we can extend Lemma 1 to the complex 
case. We need $a_{i j}^{\alpha \beta}=a_{i j}^{\beta \alpha}=a_{j i}^{\alpha \beta}$ for $\alpha, \beta=1, \ldots, m, i, j=1, \ldots, n$. Then (3.2) becomes

$$
\sum_{\alpha, \beta=1}^{m} a_{i j} \bar{\xi}_{j}^{\alpha} \xi_{i}^{\beta} \geqslant a_{0}|\vec{\xi}|^{2}
$$

The symmetry condition on the coefficients $a_{i j}^{\alpha \beta}$ insures that the left side of (3.11) is real. We also require $c^{\alpha \beta}=c^{\beta \alpha}$. Suppose $b_{0} \geqslant 0$ and $c_{0}>0$ are constants such that

$$
\left|\sum_{\alpha, \beta=1}^{m} \sum_{i=1}^{n} b_{i}^{\alpha \beta} \xi_{i}^{\beta} \bar{\eta}^{\alpha}\right| \leqslant b_{0}|\vec{\xi} \| \vec{\eta}| \text { and } \sum_{\alpha, \beta=1}^{m} c^{\alpha \beta} \bar{\eta}^{\alpha} \eta^{\beta} \leqslant-c_{0}|\vec{\eta}|^{2} \text {. }
$$

Then we can replace (3.3) with the condition

$$
\text { either } b_{i}^{\alpha \beta} \equiv c^{\alpha \beta} \equiv 0 \text { or } 4 a_{0} c_{0}>b_{0}^{2} .
$$

The argument is as follows: under (3.12) and the symmetry conditions,

$$
\begin{aligned}
\left|\sum_{\alpha, \beta=1}^{m}\left[\sum_{i, j=1}^{n} a_{i j}^{\alpha \beta} \xi_{j}^{\beta} \xi_{i}^{\beta}-\sum_{i=1}^{n} b_{i}^{\alpha \beta} \xi_{i}^{\beta} \bar{\eta}^{\alpha}-c^{\alpha \beta} \bar{\eta}^{\alpha} \eta^{\beta}\right]\right| \\
\geqslant\left|\sum_{\alpha, \beta=1}^{m}\left[\sum_{i, j=1}^{n} a_{i j}^{\alpha \beta} \bar{\xi}_{j}^{\alpha} \xi_{i}^{\beta}-c^{\alpha \beta} \bar{\eta}^{\alpha} \eta^{\beta}\right]\right|-b_{0}|\vec{\xi}||\vec{\eta}| \\
\quad=\sum_{\alpha, \beta=1}^{m}\left[\sum_{i, j=1}^{n} a_{i j}^{\alpha \beta} \bar{\xi}^{\alpha} \xi^{\beta}-c^{\alpha \beta} \bar{\eta}^{\alpha} \eta^{\beta}\right]-b_{0}|\vec{\xi}||\vec{\eta}| \\
\geqslant a_{0}|\vec{\xi}|^{2}-b_{0}|\vec{\xi}||\vec{\eta}|+c_{0}|\vec{\eta}|^{2} \\
\geqslant a_{1}\left(|\vec{\xi}|^{2}+|\vec{\eta}|^{2}\right) \quad\left(\text { if } b_{0}, c_{0} \neq 0\right)
\end{aligned}
$$

for some real $a_{1}>0$. If there are no lower order terms then (3.11) is sufficient. Conditions other than (3.12) are also possible. The proof of Lemma 1 can now be duplicated in the complex case by replacing $u^{\alpha}$ with $\bar{u}^{\alpha}$ in (3.7) and using (3.11) or (3.13) instead of (3.3) to obtain (3.8). Some care must be taken with (3.9) since we have, for $u^{\alpha}=v^{\alpha}+i w^{\alpha}$ via (2.4) and (2.13), that

$$
\int\left|\nabla u^{\alpha}\right|^{2}=\int\left|\nabla v^{\alpha}\right|^{2}+\int\left|\nabla w^{\alpha}\right|^{2}
$$

so

$$
\left\|\nabla u^{\alpha}\right\|_{0,2}^{2} \geqslant C(n)^{2}\left(\left\|v^{\alpha}\right\|_{0, p^{*}}^{2}+\left\|w^{\alpha}\right\|_{0, p^{*}}^{2}\right) \geqslant C(n)^{2} 2^{\left(p^{*}-2\right) / p^{*}}\left(\int\left|u^{\alpha}\right|^{p^{*}}\right)^{2 / p^{*}}
$$

instead of (2.4). Thus if $p \neq 2$, an additional factor, $2^{(1-\delta)\left(p^{*}-2\right) / p^{*}}$, enters into the right side of (3.6). If $p=2$ then (3.6) is unchanged.

(3) We have considered the case of a single parameter $\lambda$; however, we could also consider the analog of the generalized spectrum defined by Protter in [31]. That is, we could study the system

$$
\sum_{\alpha, \beta=1}^{m}\left[\sum_{i, j=1}^{m}\left(a_{i j}^{\alpha \beta} u_{x_{i}}^{\beta}\right)_{x_{j}}+\sum_{i=1}^{n} b_{i}^{\alpha \beta} u_{x_{i}}^{\beta}+c^{\alpha \beta} u^{\beta}\right]+\lambda^{\alpha} f^{\alpha}(x, \vec{u})=0
$$

with different values of $\lambda^{\alpha}$ in each equation. 
If we define $\vec{\lambda}=\left(\lambda^{1}, \ldots, \lambda^{m}\right)$ and use the usual Euclidean norm for $\vec{\lambda}$, replacing (3.4) with the condition $\left|u^{\alpha} f^{\alpha}(x, \vec{u})\right| \leqslant f_{1}|\vec{u}|^{q+1}$ yields

$$
\left|\sum_{\alpha=1}^{m} \lambda^{\alpha} u^{\alpha} f^{\alpha}\right| \leqslant m|\vec{\lambda}| f_{1}|\vec{u}|^{q+1}
$$

which can be used in lieu of (3.4) in (3.8). Various other definitions of $|\vec{\lambda}|$ and conditions on $\vec{f}$ are also possible. What is necessary is that

$$
\left|\sum_{\alpha=1}^{m} \lambda^{\alpha} u^{\alpha} f^{\alpha}\right| \leqslant|\vec{\lambda}| f_{2}|\vec{u}|^{q+1} \quad \text { for some } f_{2} .
$$

Using the additional symmetry conditions in Remark (2), we can allow $\vec{\lambda} \in \mathbf{C}^{m}$ also, so Lemma 1 extends to the generalized spectrum, again with minor changes.

The case of sublinear growth for $\vec{f}$ is similar to that of linear or superlinear growth, but requires somewhat different estimates. We have

Lemma 2. Suppose $\vec{u}$ satisfies (3.1) in $\Omega \subseteq \mathbf{R}^{n}, n>2$, with $\Omega$ bounded. Assume (3.3) and (3.4) hold with $0 \leqslant q<1$. Then $\vec{u}$ satisfies the inequality

$$
\|\vec{u}\|_{0, q+1}^{1-q} \leqslant|\lambda| f_{0} a_{1}^{-1} N_{1}(n, m, \Omega, q)
$$

for some constant $N_{1}(n, m, \Omega, q)>0$.

Proof. We start with (3.7) as in Lemma 1, obtain (3.8) as before, and make estimates similar to (3.9) and (3.10), using (2.9) instead of (2.8), and (2.14) instead of (2.13). Inequality (3.14) then follows immediately.

REMARKS. The remarks following Lemma 1 also apply to Lemma 2. From Lemma 1 we immediately obtain the following

TheOREM 1. Suppose $u \in W_{0}^{1,2} \cap L^{\infty}$ satisfies (3.1) on $\Omega \subseteq \mathbf{R}^{n}, n>2$, and (3.3) and (3.4) hold with $1<q \leqslant(n+2) /(n-2)$. Then

$$
\begin{aligned}
\operatorname{ess} \sup |u| & \geqslant\left(\frac{1}{|\lambda|}\right)^{1 /(q-1)}\left(\frac{1}{m|\Omega|}\right)^{1 /(q+1)}\left[\frac{a_{1} \mu_{1}(\Omega)^{\delta}}{f_{0} C(n)^{2(1-\delta)}}\right]^{1 /(q-1)} \\
& \geqslant\left(\frac{1}{|\lambda|}\right)^{1 /(q-1)}\left(\frac{1}{|\Omega|}\right)^{\gamma}\left(\frac{1}{m}\right)^{1 /(q+1)}\left[\frac{a_{1} \omega_{n}^{2 \delta / n} j_{(n-2) / n}^{2 \delta}}{f_{0} C(n)^{2(1-\delta)}}\right]^{1 /(q-1)}
\end{aligned}
$$

where $\gamma=2 \delta / n(q-1)+1 /(q+1)$ and the remaining constants are as in Lemma 1 .

ProOF. Rewriting (3.6) as

$$
\|\vec{u}\|_{0, q+1}^{q-1} \geqslant a_{1} \mu_{1}(\Omega)^{\delta} /|\lambda| f_{0} C(n)^{2(1-\delta)} m^{(q-1) /(q+1)}
$$

and taking the $1 /(q-1)$ power of both sides yields

$$
\|\vec{u}\|_{0, q+1} \geqslant\left(\frac{1}{|\lambda|}\right)^{1 /(q-1)}\left(\frac{1}{m}\right)^{1 /(q+1)}\left[\frac{a_{1} \mu_{1}(\Omega)^{\delta}}{f_{0} C(n)^{2(1-\delta)}}\right]^{1 /(q-1)} .
$$

Since $\|u\|_{0, q+1} \leqslant|\Omega|^{1 /(q+1)}$ ess sup $|u|$, inequality (3.15) follows from (3.16). 
Remarks. Theorem 1 extends to complex $u$ and $\lambda$ and to vectors $\vec{\lambda}$ in the generalized spectrum with some modifications of the hypotheses and the constants in (3.15); see the remarks following Lemma 1.

In the second line of (3.15), the Faber-Krahn bound for $\mu_{1}(\Omega)$ is used to give explicit dependence on $|\Omega|$. However, there are various other methods of estimating $\mu_{1}(\Omega)$ which sometimes give sharper results, so the first inequality may give more precise information than the second. Estimates for $\mu_{1}(\Omega)$ are discussed after Theorem 2 .

In the linear case, of course, there is no relation between $\lambda$ and the size of $|\vec{u}|$; thus Theorem 1 is a strictly nonlinear result.

Estimates somewhat similar to (3.15) for a single equation have been obtained by Schaefer and Sperb [33] for the equation $\Delta u+\lambda u^{p}=0$; their results are based on the maximum principle and thus do not extend to systems of the form (3.1). Other related results for a single equation have been obtained via the maximum principle by Payne, Schaefer, Sperb and Stakgold; these are discussed in [35]. Those results typically require weaker growth conditions on $f$, but only apply for a single equation and often require monotonicity or other conditions on $f$. Another result with similar flavor was obtained by Bandle [5] for $\Delta u+\lambda e^{u}=0$; Theorem 1 does not cover that case because of the growth conditions. Upper bounds for solutions of a single equation corresponding to (3.1) have been obtained by Gidas [14], Gidas and Spruck [16] and De Figueiredo, Lions and Nussbaum [10] by methods involving the maximum principle and symmetry arguments. For those bounds, growth conditions similar to (3.4) with $1<q \leqslant(n+2) /(n-2)$ are required. For $n=2$, upper bounds for a single equation were obtained by Turner [37].

The next theorem gives bounds for $|\lambda|$ when $f(u)$ has linear growth.

THEOREM 2. Suppose $u \in W_{0}^{1,2} \cap L^{\infty}$ satisfies (3.1), with (3.3) and (3.4) holding, and $q=1$ in (3.4). Then

$$
|\lambda| \geqslant \frac{a_{1} \mu_{1}(\Omega)}{f_{0}} \geqslant \frac{a_{1}}{f_{0}}\left(\frac{j_{(n-2) / n}^{2} \omega_{n}^{2 / n}}{|\Omega|^{2 / n}}\right) .
$$

Proof. When $q=1,(3.6)$ reduces immediately to (3.17).

REMARKS. Theorem 2 remains true without modification when $n=2$.

Theorem 2 is similar to a result of Protter [31, Theorem 1], who considers the generalized spectrum and allows complex values for the $\lambda^{\alpha}$; however, Theorem 2 can be extended to that case as is discussed in the remarks following Lemma 1. Theorem 2 allows coupling in the second order terms and nonlinearity at various places in the system; those cases are not considered in [31], but the estimates of [31] are more flexible and thus potentially sharper than that of Theorem 2.

The estimate of $\mu_{1}(\Omega)$ based on the Faber-Krahn bound can be improved in many cases. There is an extensive literature on lower bounds for eigenvalues of linear elliptic operators. Various methods of obtaining such bounds are discussed in [29, 30, 18, 26, 38, 12].

We now consider the case $0 \leqslant q<1$ as discussed in Lemma 2. In this case we must make various stronger assumptions on (3.1), and the results are not as precise. 
THEOREM 3. Suppose system (3.1) is semilinear (in other words $a_{i j}^{\alpha \beta}, b_{i}^{\alpha \beta}$ and $c^{\alpha \beta}$ are independent of $\vec{u}$ ) with smooth coefficients (being of class $C^{2}$ is sufficient, but weaker conditions are possible) and $\vec{f}(x, \vec{u})$ satisfies

$$
|\vec{f}(x, \vec{u})| \leqslant f_{1}|\vec{u}|^{q}, \quad 0 \leqslant q<1 .
$$

If $\vec{u}$ is a classical solution of (3.1), then

$$
\sup |\vec{u}| \leqslant C|\lambda|^{1 /(1-q)},
$$

where $C$ depends on $f_{1}, a_{i j}^{\alpha \beta}, b_{i}^{\alpha \beta}, c^{\alpha \beta}, \Omega, m, n$ and $q$.

Proof. If we define the operator $A_{p}$ on $W^{2, p} \cap W_{0}^{1, p}$ by

$$
\left(A_{p} \vec{v}\right)^{\alpha}=\sum_{\beta=1}^{m}\left[\sum_{i, j=1}^{n}\left(a_{i j}^{\alpha \beta}(x) v_{x_{i}}^{\beta}\right)_{x_{j}}+\sum_{i=1}^{n} b_{i}^{\alpha \beta}(x) v_{x_{i}}^{\beta}+c^{\alpha \beta}(x) v^{\beta}\right], \quad \alpha=1, \ldots, m,
$$

then, by (3.2), $A_{p}$ is elliptic and satisfies the hypotheses for the a priori estimates of [2]. Further, (3.3) implies that the nullspace of $A_{p}$ is zero if $p \geqslant 2$; if $p \geqslant 2$ the conjugate index, $p^{\prime}$, of $p$ satisfies $p^{\prime} \leqslant p$, so, by (2.7), $L^{p^{\prime}} \subseteq L^{p}$, and for $A_{p} \vec{u}=0$ we can use $\vec{u}$ itself as a test function in the equation

$$
\int \vec{v} \cdot A_{p} \vec{u}=0
$$

Thus, by (3.3), we have $\int|\nabla \vec{u}|^{2}=0$, which, together with $\vec{u} \in W_{0}^{1, p}$, implies $\vec{u}=0$ a.e. Hence for $p \geqslant 2$, nullspace $A_{p}=\{0\}$, so we can apply the estimates of [2] to obtain

$$
\|\vec{u}\|_{2, p} \leqslant C_{p}\left\|A_{p} \vec{u}\right\|_{0, p} .
$$

(Results of this sort are discussed for a single equation in [13]; see also [34]. The case of systems is similar.)

By Lemma 2, we have

$$
\|\vec{u}\|_{0,1+q} \leqslant C|\lambda|^{1 /(q-1)}
$$

Then (3.18) implies

$$
\begin{aligned}
\|\vec{f}(\alpha, \vec{u})\|_{0,1+1 / q} & =\left(\int|\vec{f}|^{1+1 / q}\right)^{q /(1+q)} \leqslant\left(\int f_{1}^{1+1 / q}|\vec{u}|^{1+q}\right)^{q /(1+q)} \\
& \leqslant f_{1}\|\vec{u}\|_{0,1+q}^{q} \leqslant C|\lambda|^{q /(1-q)} .
\end{aligned}
$$

Let $p_{0}=1+1 / q \geqslant 2$; then combining (3.1), (3.20) and (3.22) yields

$$
\|\vec{u}\|_{2, p_{0}}<|\lambda| C_{p_{0}}\|\vec{f}(x, \vec{u})\|_{0, p_{0}} \leqslant C|\lambda|^{1+q /(1-q)}=C|\lambda|^{1 /(1-q)} .
$$

If $2 p_{0}>n$ then (3.23) together with (2.12) yield (3.19) immediately. If $2 p_{0}<n$, then by $(2.10)$ we have

$$
\|u\|_{0, n p_{0} /\left(n-2 p_{0}\right)} \leqslant C|\lambda|^{1 /(1-q)} .
$$

Let $p_{1}=n p_{0} / q\left(n-2 p_{0}\right)$; then as in (3.22) and (3.23),

$$
\|\vec{f}(x, \vec{u})\|_{0, p_{1}} \leqslant C|\lambda|^{q /(1-q)}
$$


and

$$
\|\vec{u}\|_{2, p_{1}} \leqslant C|\lambda|^{1 /(1-q)} .
$$

If we define $p_{l+1}=n p_{l} / q\left(n-2 p_{l}\right)$, then, proceeding as above, we have by induction,

$$
\|\vec{u}\|_{2, p,} \leqslant C|\lambda|^{1 /(1-q)} .
$$

Also, $p_{0}>2$, and if $p_{l}>2, p_{l+1}>n p_{l} / q(n-2)>[n /(n-2)] p_{l}$. Since $n /(n-2)$ $>1$, it follows that $p_{l}>[n /(n-2)]^{l} p_{0}$, so by iteration we eventually obtain $2 p_{l}>n$. Now (2.12) can be applied to (3.26) to yield (3.19). (Since we are working with a system, the constants depend on $m$. See the remarks at the end of $\S 2$.)

REMARKS. The estimate in (3.19) is rough; it primarily gives an a priori bound in terms of $|\lambda|$. In specific cases it may be possible to refine the method to give more precise results. In [33] much more precise estimates based on the maximum principle are obtained for $\Delta u+\lambda u^{p}=0$; however, (3.1) may not satisfy a maximum principle, and no monotonicity conditions are required in Theorem 3.

As noted in the remarks following Theorem 2, that theorem holds with no changes for $n=2$. Theorem 3 does also; in fact, for $n=2, W^{2,1+1 / q}$ embeds in $C^{0}(\Omega)$, so we can use (2.12) instead of (2.10), and iteration past (3.23) is unnecessary. Theorem 1 can be extended to the case $n=2$, but it requires certain modifications. The constant $C(n)$ in (3.16) must be replaced by a constant depending on $q$, and certain other changes are necessary in the estimates; on the other hand, any $q>1$ may be used in (3.4).

We have

THEOREM 4. Suppose $u \in W_{0}^{1,2} \cap L^{\infty}$ satisfies (3.1), with (3.3) and (3.4) satisfied, $q>1$, and $n=2$. Then

$$
\begin{aligned}
\operatorname{ess} \sup |\vec{u}| \geqslant & (1 /|\lambda|)^{1 /(q-1)}(1 / m|\Omega|)^{1 /(q+1)} \\
& \cdot\left[a_{1} \mu_{1}(\Omega) / f_{0} M(p)^{2}\left(1+\mu_{1}(\Omega)\right)\right]^{1 /(q-1)},
\end{aligned}
$$

where $M(p)$ is the constant in (2.11) for the embedding of $W_{0}^{1,2}$ in $L^{p}, p=q+1$.

(Since we consider only $\vec{u} \in W_{0}^{1,2}$, we can choose $M(p)$ independently of $\Omega$; see the discussion after (2.11).)

Proof. As in Lemma 1, we have

$$
a_{1} \sum_{\alpha=1}^{m} \int\left|\nabla u^{\alpha}\right|^{2} \leqslant|\lambda| f_{0}\|u\|_{0, p}^{p}
$$

where $p=1+q$. From (2.2) for $\tau \in[0,1]$ we have

$$
\sum_{\alpha=1}^{m} \int\left|\nabla u^{\alpha}\right|^{2} \geqslant \sum_{\alpha=1}^{m}(1-\tau) \int\left|\nabla u^{\alpha}\right|^{2}+\mu_{1}(\Omega) \tau \int\left|u^{\alpha}\right|^{2}
$$

Choosing $\tau=1 /\left(1+\mu_{1}(\Omega)\right)$, so that $\mu_{1}(\Omega) \tau=1-\tau$, yields

$$
\sum_{\alpha=1}^{m}\left|\nabla u^{\alpha}\right|^{2} \geqslant \frac{\mu_{1}(\Omega)}{1+\mu_{1}(\Omega)} \sum_{\alpha=1}^{m}\left\|u^{\alpha}\right\|_{1,2}^{2} \text {. }
$$


By (2.11) and the remarks following, $\left\|u^{\alpha}\right\|_{0, p} \leqslant M(p)\left\|u^{\alpha}\right\|_{1,2}$ for some constant $M(p)$; applying (2.13) and (3.28) yields from (3.8)

$$
\left[\mu_{1}(\Omega) /\left(1+\mu_{1}(\Omega)\right)\right]\|\vec{u}\|_{0, p}^{2} \leqslant|\lambda| f_{0} a_{1}^{-1} M(p)^{2} m^{(p-2) / p}\|\vec{u}\|_{0, p}^{p} .
$$

Inequality (3.27) follows immediately from (3.30) as in the proof of Theorem 1.

REMARKs. The discussion following Lemma 1 also applies to Theorem 4.

Thus far we have imposed the coercivity condition (3.3), but our results remain true under condition (3.2) alone if $|\Omega|$ is sufficiently small. Since we assume $b_{i}^{\alpha \beta}$ and $c^{\alpha \beta}$ are bounded, there are constants $b_{1}, c_{1}$ such that

$$
\left|\sum_{\alpha, \beta=1}^{m} \sum_{i=1}^{n} b_{i}^{\alpha \beta} \xi_{i}^{\beta} \eta^{\alpha}\right| \leqslant b_{1}|\xi \| \eta| \text { and }\left|\sum_{\alpha, \beta=1}^{m} c^{\alpha \beta} \eta^{\alpha} \eta^{\beta}\right| \leqslant c_{1}|\eta|^{2} .
$$

Thus, for any $\varepsilon>0$, we have, by (3.2),

$$
\begin{gathered}
\left|\int \sum_{\alpha, \beta=1}^{m}\left[\sum_{i, j=1}^{n} a_{i j}^{\alpha \beta} u_{j}^{\alpha} u_{i}^{\beta}-\sum_{i=1}^{n} b_{i}^{\alpha \beta} u^{\alpha} u_{i}^{\beta}-c^{\alpha \beta} u^{\alpha} u^{\beta}\right]\right| \\
\geqslant \int\left[a_{0} \sum_{\alpha=1}^{m}\left|\nabla u^{\alpha}\right|^{2}-b_{1}\left(\sum_{\alpha=1}^{m}\left|\nabla u^{\alpha}\right|^{2}\right)^{1 / 2}|\vec{u}|-c_{1}|\vec{u}|^{2}\right] \\
\geqslant\left(a_{0}-\frac{\varepsilon b_{1}}{2}\right) \int \sum_{\alpha=1}^{m}\left|\nabla u^{\alpha}\right|^{2}-\left(\frac{b_{1}}{2 \varepsilon}+c_{1}\right) \int|\vec{u}|^{2} .
\end{gathered}
$$

But by (2.2),

$$
\int|\vec{u}|^{2} \leqslant \frac{|\Omega|^{2 / n}}{j_{(n-2) / n}^{2} \omega_{n}^{2}} \int \sum_{a=1}^{m}\left|\nabla u^{\alpha}\right|^{2}
$$

Thus we have

$$
\begin{aligned}
\left|\int \sum_{\alpha, \beta=1}^{m}\left[\sum_{i, j=1}^{n} a_{i j}^{\alpha \beta} u_{x_{j}}^{\alpha} u_{x_{i}}^{\beta}-\sum_{i=1}^{n} b_{i}^{\alpha \beta} u^{\alpha} u_{x_{i}}^{\beta}-c^{\alpha \beta} u^{\alpha} u^{\beta}\right]\right| \\
\geqslant\left[a_{0}-\frac{\varepsilon b_{1}}{2}-\left(\frac{|\Omega|^{2 / n}}{j_{(n-2) / n}^{2} \omega_{n}^{2}}\right)\left(\frac{b_{1}}{2 \varepsilon}+c_{1}\right)\right] \int \sum_{\alpha=1}^{m}\left|\nabla u^{\alpha}\right|^{2} .
\end{aligned}
$$

For any $\varepsilon_{0}$ with $0<\varepsilon_{0}<a_{0}$ we can choose $\varepsilon=2 \varepsilon_{0} / b_{1}$ and then require $|\Omega|$ to be small enough that the constant on the right side of (3.32) is positive. In that case we can use (3.32) to obtain estimates analogous to (3.8). Thus we have

THEOREM 5. The results of this section remain true under (3.2) instead of (3.3) if $a_{1}$ is replaced by the constant

$$
a_{2}\left(\Omega, \varepsilon_{0}\right)=a_{0}-\varepsilon_{0}-\left(\frac{|\Omega|^{2 / n}}{j_{(n-) / n}^{2} \omega_{n}^{2}}\right)\left(\frac{b_{1}^{2}}{4 \varepsilon_{0}}+c_{1}\right),
$$

where $a_{0}>\varepsilon_{0}>0$ and $|\Omega|$ is small enough to make $a_{2}\left(\Omega, \varepsilon_{0}\right)>0$. In particular, for any $\varepsilon>0$ we can choose $\varepsilon_{0}=\varepsilon / 2$ and then find a value $V(\varepsilon)>0$ such that for $|\Omega|<V(\varepsilon), a_{2}(\Omega, \varepsilon)>a_{0}-\varepsilon$. 
Remarks. Theorem 5 extends to complex $\lambda$ and to the generalized spectrum for (3.1) under the same type of additional assumptions and modifications needed for the other results of this section; see the remarks following Lemma 1. A result analogous to that obtained by applying Theorem 5 to Theorem 2 for the generalized spectrum was proved by Protter [31, Theorem 2] in the case of a linear system with no coupling in the second order terms.

4. Higher order problems. In this section we give a brief discussion of how the results of the previous section extend to elliptic systems of order $2 k, k>1$. For simplicity we consider the system

$$
\sum_{\beta=1}^{m} \sum_{|\sigma|=|\tau|=k}\left(a_{\sigma \tau}^{\alpha \beta}(x, \vec{u}) u_{x_{\sigma}}^{\beta}\right)_{x_{\tau}}+c^{\alpha \beta}(x, \vec{u}) u^{\beta}=\lambda f^{\alpha}(x, \vec{u}),
$$

where $\sigma, \tau$ are multi-indices. We require that $u \in W_{0}^{k, 2} \cap L^{\infty}$, which imposes the usual form of Dirichlet boundary conditions for weak solutions on $\vec{u}$, and that all coefficients of (4.1) are bounded and measurable. Analogous to (3.2) and (3.3) we require

$$
\sum_{\alpha, \beta=1}^{m}\left[\sum_{|\sigma|=|\tau|=k} a_{\sigma \tau}^{\alpha \beta} \xi_{\tau}^{\alpha} \xi_{\sigma}^{\beta}+(-1)^{k} c^{\alpha \beta} \eta^{\alpha} \eta^{\beta}\right] \geqslant a_{0}\left(|\vec{\xi}|^{2}+|\vec{\eta}|^{2}\right)
$$

for all $x \in \Omega, \vec{u} \in \mathbf{R}^{m}, \xi \in \mathbf{R}^{m C(n, k)}$ and $\eta \in \mathbf{R}^{m}$, where

$$
C(n, k)=(n+k-1) ! / k !(n-1) ! \text {. }
$$

We also need the following inequality, which is a consequence of Theorem 4.13 of [1]:

$$
\|V\|_{k, 2}^{2} \leqslant M_{3}(n, k)\left[\sum_{|\sigma|=k}\left\|V_{x^{\sigma}}\right\|_{0,2}^{2}+\|V\|_{0,2}^{2}\right] .
$$

Condition (3.4) on $\vec{f}(x, u)$ will be imposed as before. Analogous to the proofs of Lemmas 1 and 2, we have

$$
\int \sum_{\alpha, \beta=1}^{m}\left[\sum_{|\sigma|=|\tau|=k} a_{\sigma \tau}^{\alpha \beta} u_{x^{o}}^{\alpha} u_{x^{\tau}}^{\beta}+(-1)^{k} c^{\alpha \beta} u^{\alpha} u^{\beta}\right] \leqslant|\lambda| f_{0} \int|\vec{u}|^{1+q},
$$

which leads, via (4.2) and (4.3), to

$$
\|\vec{u}\|_{k, 2}^{2} \leqslant|\lambda| a_{0}^{-1} M_{3}(n, k)\|\vec{u}\|_{0, p}^{p}
$$

where $p=1+q$. Note that (4.3) allows us to handle terms of order greater than zero but less than $2 k$ in (4.1) if their coefficients are sufficiently small compared to $a_{0}$ or satisfy the appropriate sign conditions. The details are omitted in the interest of brevity.

From (4.5) we proceed as in $\S 3$, with the details depending on $n$ and $p$. If $2<2 k<n$ and $2 \leqslant p \leqslant 2 n /(n-2 k)$, or if $2 k=n$ and $p \geqslant 2$, we can apply (2.10) or (2.11) (with constant independent of $\Omega$; see the comments following (2.12)) and (2.13) to obtain

$$
\|\vec{u}\|_{0, p}^{2} \leqslant m^{(p-2) / 2} \sum_{\alpha=1}^{m}\left\|u^{\alpha}\right\|_{0, p}^{2} \leqslant|\lambda| m^{(p-2) / 2} a_{0}^{-1} f_{0} M_{4}(n, k, p)\|\vec{u}\|_{0, p}^{p}
$$


or

$$
1 \leqslant|\lambda| m^{(p-2) / p} a_{0}^{-1} f_{0} M_{4}(n, k, p)\|\vec{u}\|_{0, p}^{p-2} .
$$

If $p>2$ then, as in Theorem 1 ,

$$
\text { ess sup }|\vec{u}| \geqslant\left(\frac{1}{|\lambda|}\right)^{1 / q-1}\left(\frac{1}{m|\Omega|}\right)^{1 /(q+1)}\left[\frac{a_{0}}{f_{1} M_{4}(n, k, p)}\right]^{1 / q-1} .
$$

If $p=2$ we get, as in Theorem 2, a lower bound for $|\lambda|$. (The estimate is crude; it could be sharpened by obtaining estimates for the first eigenvalue of

$$
\sum_{|\sigma|=k} u_{x^{2 \sigma}}+\mu u=0
$$

and using them instead of the Sobolev inequality to obtain (4.6) from (4.4). Also, with such an approach (4.2) could be weakened by replacing the right side with $a_{0}|\vec{\xi}|^{2}$.) The case $p=2$ can be handled in much the same way independently of $n$; if $p>2$ and $n<2 k$ we can make the following estimate using (2.12) and the comments preceding (2.14):

$$
\sup |\vec{u}|^{2} \leqslant|\lambda| a_{0}^{-1} f_{0} M_{5}(m, n, k)(\sup |\vec{u}|)^{p}|\Omega| .
$$

Inequality (4.9) then yields results analogous to (4.8).

If $1 \leqslant p<2$ we proceed as in Lemma 2 and Theorem 3. For $2 k \leqslant n$ we have, in lieu of (4.6),

$$
\|\vec{u}\|_{0, p}^{2} \leqslant|\lambda| a_{0}^{-1} f_{0} M_{6}(n, m, k, \Omega ; p)\|\vec{u}\|_{0, p}^{p}
$$

or

$$
\|\vec{u}\|_{0, q+1}^{1-q} \leqslant|\lambda| a_{0}^{-1} f_{0} M_{6}(n, m, k, \Omega, p) .
$$

Inequality (4.10) is analogous to (3.14) and can be used in the same way to obtain a result similar to Theorem 3 . As in Theorem 3 we must assume the coefficients of (4.1) are smooth and independent of $\vec{u}$.

If $2 k>n$ then we can use (2.12) and the arguments justifying (2.14) to immediately obtain

$$
(\sup |\vec{u}|)^{2} \leqslant|\lambda| a_{0}^{-1} f_{0} M_{7}(n, m, k, \Omega, p)|\Omega|(\sup |\dot{\vec{u}}|)^{p}
$$

or

$$
(\sup |\vec{u}|) \leqslant\left[|\lambda| a_{0}^{-1} f_{0} M_{7}(n, m, k, \Omega, p)\right]^{1 /(1-q)} .
$$

Thus the methods of $\S 3$ also apply with minor modifications to the case of higher order systems.

\section{REFERENCES}

1. R. Adams, Sobolev spaces, Academic Press, New York, 1975.

2. S. Agmon, A. Douglis and L. Nirenberg, Estimates near the boundary for solutions of elliptic partial differential equations satisfying general boundary conditions. II, Comm. Pure Appl. Math. 17 (1964), 35-92.

3. H. Amann and M. Crandall, On some existence theorems for semi-linear elliptic equations, Indiana Univ. Math. J. 27 (1978), 779-790. 
4. A. Ambrosetti and P. Rabinowitz, Dual variational methods in critical point theory and applications, J. Funct. Anal. 14 (1973), 349-381.

5. C. Bandle, Isoperimetric inequalities for a nonlinear eigenvalue problem, Proc. Amer. Math. Soc. 56 (1976), 243-246.

6. Isoperimetric inequalities and applications, Monographs Stud. Math., no. 7, Pitman, Boston, Mass., 1980.

7. J. Barta, Sur la vibration fondamentale d' une membrane, C. R. Acad. Sci. Paris Sér. 204 (1937), 472.

8. M. Berger, An eigenvalue problem for nonlinear partial differential equations, Trans. Amer. Math. Soc. 120 (1965), 145-184.

9. F. Browder, Infinite dimensional manifolds and non-linear elliptic eigenvalue problems, Ann. of Math. (2) 82 (1965), 459-477.

10. D. De Figueiredo, P.-L. Lions and R. Nussbaum, A priori estimates and existence of positive solutions of semilinear elliptic equations, J. Math. Pures Appl. (9) 61 (1982), 41-63.

11. C. Faber, Beweis, dass unter allen homogenen Membranen von gleicher Flache und gleicher Spannung die kreisformige tiefsten Grundton gibt, Sitzungsber. Bayer. Akad. Wiss. Math.-Phys., Munich, 1923, pp. 169-172.

12. G. Fichera, Il calcolo degli autovalori, Boll. Un. Mat. Ital. (4) 1 (1968), 33-95.

13. A. Friedman, Partial differential equations, Krieger, New York, 1976.

14. B. Gidas, Symmetry properties and isolated singularities of positive solutions of nonlinear elliptic equations, Conference Nonlinear Partial Differential Equations in Engineering and Applied Science (R. Stenberg, A. Kalinowski and J. Papadakis, eds.), Dekker, New York, 1980.

15. B. Gidas, W.-M. Ni and L. Nirenberg, Symmetry and related properties via the maximum principle, Comm. Math. Phys. 68 (1979), 209-243.

16. B. Gidas and J. Spruck, A priori bounds for positive solutions of nonlinear elliptic equations, Comm. Partial Differential Equations 6 (1981), 883-901.

17. D. Gilbarg and N. Trudinger, Elliptic partial differential equations of second order, Springer-Verlag, Berlin and New York, 1977.

18. J. Hersch, Physical interpretation and strengthening of $M$. H. Protter's method for vibrating nonhomogeneous membranes; its analogue for Schrödinger's equation, Pacific. J. Math. 11 (1962), 971-980.

19. E. Krahn, Über eine van Rayleigh formulierte Minimaleigenschaft des Kreises, Math. Ann. 94 (1925). $97-100$.

20. . Uber Minimaleigenschaften der Kugel in drei und mehr Dimensionen, Acta Comm. Univ. Tartu (Dorpat) A9 (1926), 1-44.

21. R. Kramer, Sub- and super-solutions of quasilinear elliptic boundary value problems, J. Differential Equations 28 (1978), 278-283.

22. N. Levinson, Positive eigenfunctions for $\Delta u+\lambda f(u)=0$. Arch. Rational Mech. Anal. 11 (1962), $258-272$.

23. R. Osserman, The isoperimetric inequality, Bull. Amer. Math. Soc. 84 (1978), 1182-1238.

24. L. Payne, Isoperimetric inequalities and their applications, SIAM Rev. 9 (1967), 453-488.

25. L. Payne and I. Stakgold, Nonlinear problems in nuclear reactor analysis, Nonlinear Problems in the Physical Sciences and Biology (I. Stakgold, D. Joseph and D. Sattinger, eds.), Lecture Notes in Math., vol. 322. Springer-Verlag, Berlin and New York, 1973.

26. L. Payne and H. Weinberger, Lower bounds for vibration frequencies of elastically supported membranes and plates, J. Soc. Indust. Appl. Math. 5 (1957), 171-182.

27. S. Pohozaev, Eigenfunctions of the equation $\Delta u+\lambda f(u)=0$, Dokl. Akad. Nauk SSSR 165 (1965), 36-39; English transl., Soviet Math. Dokl. 6 (1965), 1408-1411.

28. G. Pólya and G. Szegö, Isoperimetric inequalities in mathematical physics, Princeton Univ. Press, Princeton, N. J., 1951.

29. M. Protter, Vibrations of a nonhomogeneous membrane, Pacific J. Math. 9 (1959), 1249-1255.

30. Lower bounds for the first eigenvalue of elliptic equations, Ann. of Math. (2) 71 (1960), 423-444.

31. The generalized spectrum of second order elliptic slytems, Rocky Mountain J. Math. 9 (1979), 503-518.

32. M. Protter and H. Weinberger, On the spectrum of general second order operators, Bull. Amer. Math. Soc. 72 (1966), 251-255.

33. P. Schaefer and R. Sperb, Maximum principles and bounds in some inhomogeneous elliptic boundary value problems, SIAM J. Math. Anal. 8 (1977), 871-878. 
34. C. Simader, On Dirichlet's boundary value problem, Lecture Notes in Math., vol. 268, Springer-Verlag, Berlin and New York, 1972.

35. R. Sperb, Maximum principles and their applications, Academic Press, New York, 1981.

36. G. Talenti, Best constant in Sobolev inequality, Ann. Mat. Pura Appl. (4) 110 (1976), 353-372.

37. R. Turner, A priori bounds for positive solutions of nonlinear elliptic equations in two variables, Duke Math. J. 41 (1974), 759-774.

38. H. Weinberger, Upper and lower bounds for eigenvalues by finite difference methods, Comm. Pure Appl. Math 9 (1956), 613-623.

Department of Mathematics and Computer Science, University of Miami, Coral Gables, FLORIDA 33124 